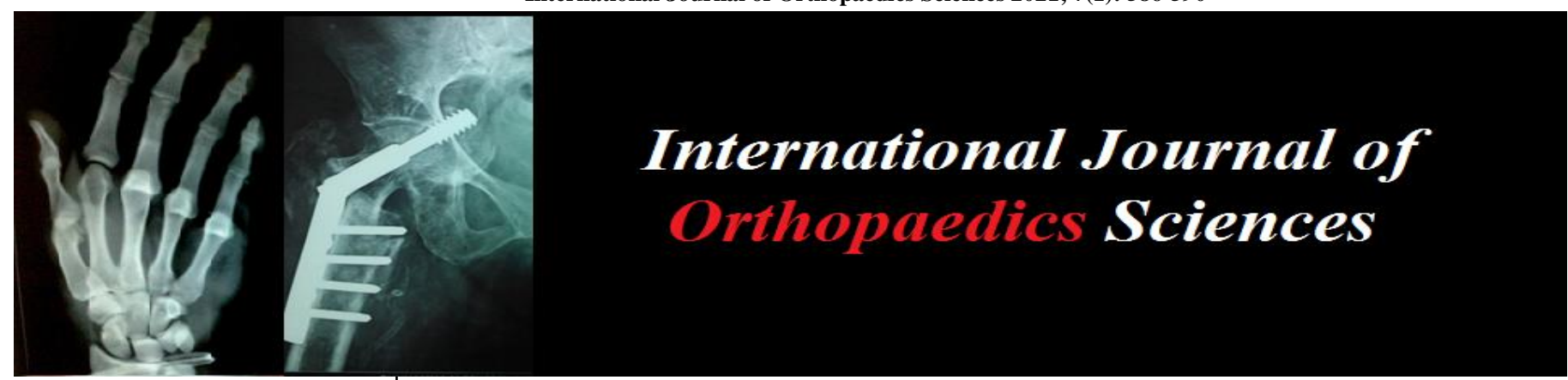

E-ISSN: 2395-1958

P-ISSN: 2706-6630

IJOS 2021; 7(1): 586-590

(C) 2021 IJOS

www.orthopaper.com

Received: 29-10-2020

Accepted: 15-12-2020

\section{Pranav Kothiyal}

Assistant professor, Department of Orthopaedics, SGRRIMHS,

Dehradun, Uttarakhand India

\section{Kunal Vij}

Associate Professor, Department of Orthopaedics, SGRRIMHS,

Dehradun, Uttarakhand India

\section{Puneet Gupta}

Professor and head, Department of Orthopaedics, SGRRIMHS

Dehradun, Uttarakhand India

Tarun Bagla

Senior resident Department of Orthopaedics, SGRRIMHS

Dehradun, Uttarakhand India

Corresponding Author: Kunal Vij

Associate Professor, Department of Orthopaedics, SGRRIMHS,

Dehradun, Uttarakhand India

\section{Outcome of Distal radius fracture managed by MIPO - A case series}

\section{Pranav Kothiyal, Kunal Vij, Puneet Gupta and Tarun Bagla}

DOI: https://doi.org/10.22271/ortho.2021.v7.i1j.2543

\section{Abstract}

Introduction: Various treatment options for management of distal radius fracture include close reduction and application of cast, $\mathrm{K}$ wire fixation, external fixator application and open reduction and plating.

The current study is aimed to assess the outcome of distal end radius fracture managed by minimally invasive plate osteosynthesis by evaluation of clinical results, radiological features and functional outcome.

Materials and Methods: This was a prospective study conducted in Orthopedics department of SGRRIMHS, Dehradun 80 patients were enrolled. Evaluation was done on the basis of clinicoradiological and functional assessment. The parameters were measured preoperatively and on 4, 12 and 24 weeks. Chi square test was used to compare proportions and T-test was used to compare means of groups. $P$-value $<0.05$ was considered significant.

Results: Mean age of participants was $42.55 \pm 19.5$ years. Mean duration of surgery was 76.05 minutes.

Mean post op Radial inclination was 18.10 degree, Mean post op Volar tilt was 10.45 degree, Mean post op Radial height was $9.25 \mathrm{~mm}$. Mean Wrist flexion at final follow up achieved was 51 degree and Mean Wrist extension was found to be 42.5 degree. Mean percentage of Grip Strength was $81.8 \%$.DASH score at final follow up was found to be 7.2.

Conclusion: Minimally invasive plate osteosynthesis can be utilised for extra articular distal radius fracture and intra articular fractures with minimal communition as it avoids extensive soft tissue dissection, preserve pronator quadratus and periosteal blood supply and provides rigid fixation, allows early mobilisation and better clinical, radiological and functional outcomes.

Keywords: distal radius fracture; MIPO; minimally invasive plate osteosynthesis; fracture management

\section{Introduction}

Fracture of distal end radius is one of the common fractures encountered by orthopaedic surgeons in their daily practice. The conservative treatment option include manipulation for close reduction and application of cast under regional or general anaesthesia. However, the results are often unsatisfactory with restricted function and disabilities. The minimal invasive procedures include manipulation for close reduction and percutaneous $\mathrm{k}$ wire fixation or external fixator application. Several studies have reported good anatomical and functional results of distal radius fractures treated with close reduction with $\mathrm{k}$ wire fixation and external fixation but also described high incidence of pin tract infection, cosmetic deformity, and nerve injury ${ }^{[1]}$. Traditional open reduction and internal fixation often results in extensive soft tissue dissection and periosteal stripping and may be associated with high rates of infection, delayed union and non-union ${ }^{[2,3]}$. Literature suggests that the recently emerged technique of minimally invasive plate osteosynthesis (MIPO) can minimize soft tissue stripping and damage to the vascularity of the bone fragments ${ }^{[4]}$. It can lead to a reduction in complications caused by conventional open treatment. The minimally invasive plate osteosynthesis is widely used in cases of lower limb fractures, but its application in upper limb fractures is not in frequent use.

The current study is aimed to assess the outcome of distal end radius fracture managed by minimally invasive plate osteosynthesis by evaluation of clinical results, radiological features and functional outcome. 


\section{Materials and Methods}

This was a prospective study conducted in Orthopedics department of SGRRIMHS and SMIH, Dehradun. Patients presenting with distal end radius fracture in SMI hospital emergency or OPD from April 2017 to September 2019 were included in this study. Total 80 patients were enrolled in this study.

Patients having age $\geq 18$ year with extra articular and minimally comminuted intra-articular fractures were included in the study.

Patients having age $<18$ year, open fracture, severely comminuted intra-articular fracture, fracture $5 \mathrm{~cm}$ proximal to articular surface, ipsilateral fracture of other upper limb bones, pathological fracture, previous history of radius fracture, preexisting stiffness of ipsilateral joints, dorsal Barton or severe dorsal comminution were excluded from the study.

\section{Surgical Technique}

The patient was placed supine on the OT table and brachial plexus block was given. The first step was to achieved closed reduction by traction and counter traction method. Once desirable reduction was obtained and confirmed under fluoroscopy the fracture was provisionally held with the help of two $\mathrm{k}$ wires. A transverse incision was given at the proximal wrist crease approx. $2 \mathrm{~cm}$ in length and a interval between flexor carpi radials and radial artery was made. The tendon of flexor pollicis longs was identified and retracted medially following which the belly of pronator quadratus was visualised. The PQ was split transversely at the distal edge and the anterior cortex of distal radius was exposed. If the PQ is already found to be injured then no additional incision was given and care was taken to preserve the remaining attachment of PQ.

Showing distal transverse and proximal longitudinal incision. (b) Showing extra periosteal, submuscular tunnel
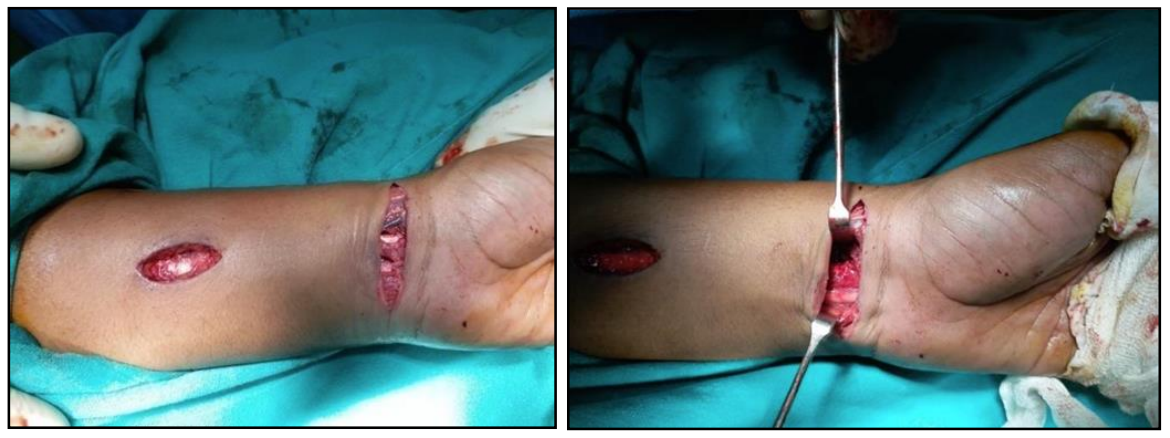

Pre op Radiographs and Post op Radiographs after doing plate fixation by MIPO and depicting corrected radiological parameters
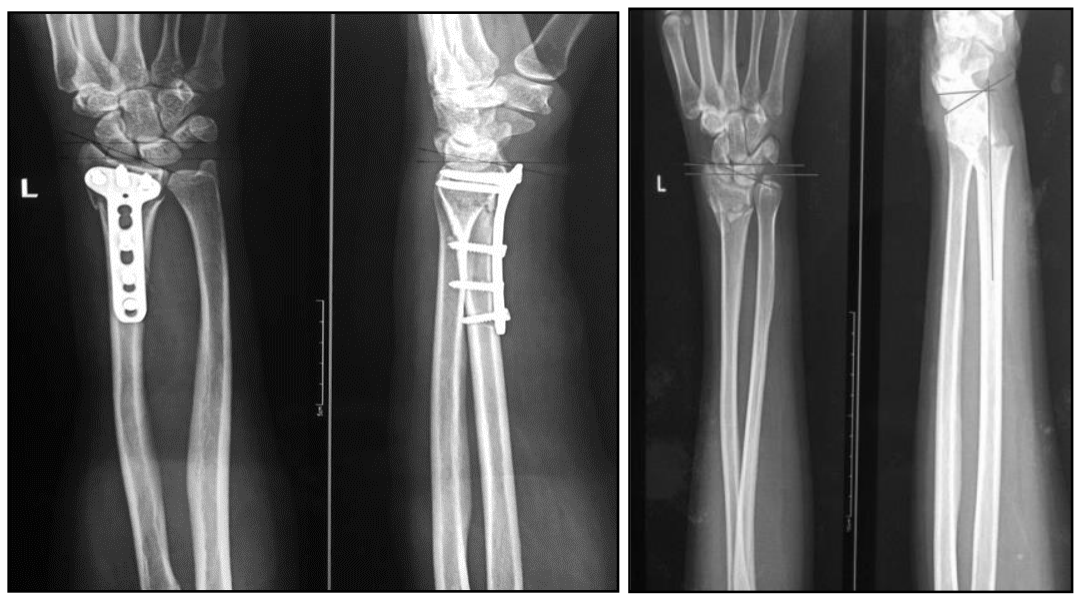

Functional results of left wrist at final follow up showing good dorsiflexion, (b)palmar flexion, (c) supination and the healed scar mark (d) and pronation

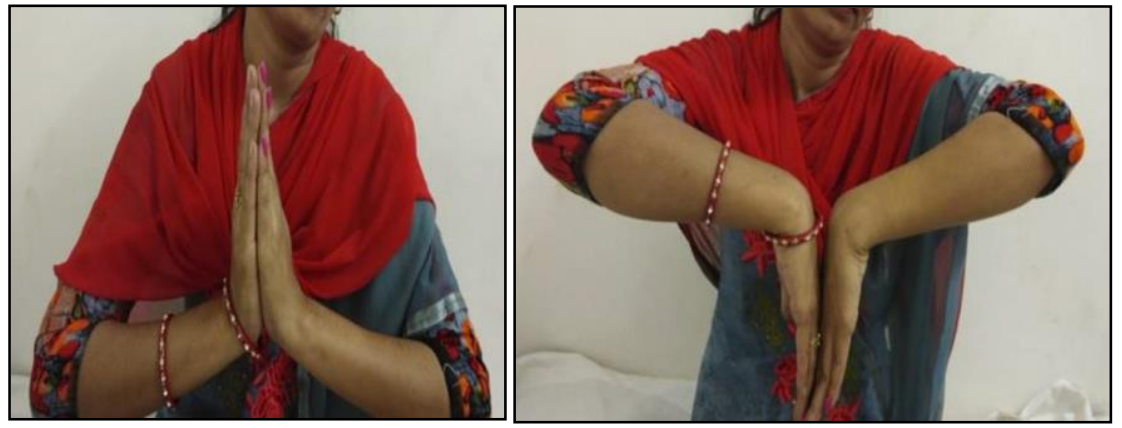




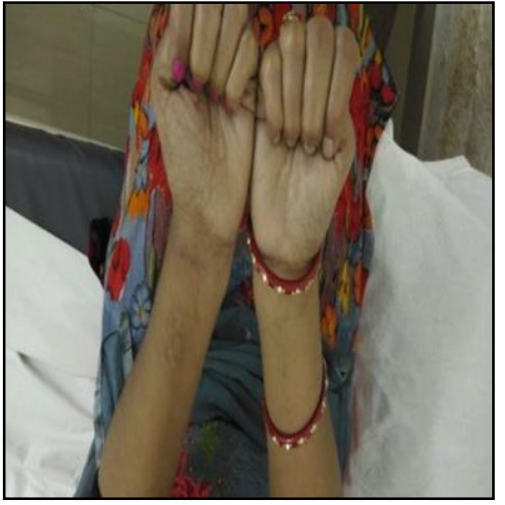

A submuscular extraperiosteal tunnel was made beneath the pronator quadratus and underlying periosteum and precontured distal radius T-Plate was inserted. The T-plate was held to the bone temporarily with a $1.5 \mathrm{~mm} \mathrm{k}$ wire passed through one of the distal hole.

Another $2 \mathrm{~cm}$ long longitudinal incision was made corresponding to the proximal end of the plate along the medial border of the brachioradialis in the mid portion of forearm. The proximal part of the plate was also held to the bone by another $1.5 \mathrm{~mm} \mathrm{k}$ wire keeping the plate centrally over the anterior surface of radius. The final reduction at the fracture site and the placement of the plate was confirmed on fluoroscopy in AP and LAT views. If any malreduction was still present, it was corrected manually.

Locking screws were placed distally first and the fracture was fixed and the temporary $\mathrm{k}$ wires were removed. Proximally a cortical screw was placed first at the proximal end of the plate, the purpose of which was to regain the volar tilt. Care was taken to prevent any inarticular placement of the distal screws and protrusion of distal screws in the extensor compartment. Wound was thoroughly washed and pronator quadratus was repaired followed by closure of the wound.

\section{Post-Op Care}

After surgery the forearm was kept in elevation and active/passive movements of fingers were started from first post op day. Immediate physiotherapy was started in majority of the patients however certain factors like the communition at fracture site, osteoporosis, the stability of fixation were kept in mind.

The fractures that were communited or in old age patients with osteoporosis the limb was immobilised in a posterior slab till the sutures were removed.

Finger, elbow and shoulder range of motion exercises were started from first post op day.

Wrist dorsiflexion and palmar flexion was started according to the tolerance of the patient. It was done immediately in young patients with stable fracture pattern and rigid fixation while in patients having osteoporosis or complex fracture patterns wrist movements were started delayed after suture removal. During the stay of patients in the hospital they were constantly monitored for compliance to physiotherapy and any immediate post op complications.

Assesment of the patients were done on various parameters. Factors regarding surgical techniques and intraoperative variables were evaluated. Bone union was evaluated by radiographs at every followup and functional outcomes were monitored by DASH score, Grip strength and Green and O'brien score.

Operative time was recorded from the first incision that was given till the last suture, fluoroscopy time was recorded from its first shot till the last one and the blood loss was estimated

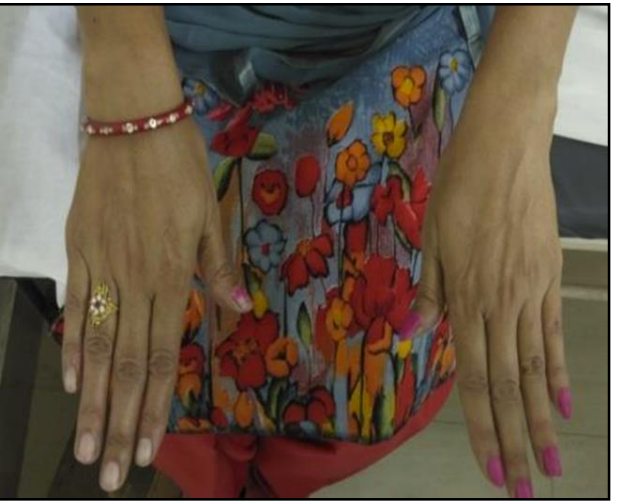

by the number of sponges used and the amount of solution in the suction container at the end of surgery.

Radiographic assessment was done on the basis of Radial height, Radial inclination, volar tilt. These were measured on pre-operative radiographic films and post-operative films and were compared. These parameters were also measured on subsequent follow ups at 4,12 and 24 weeks and compared with the previous films to look for any collapse at fracture site.

The union at fracture site was also seen on X-rays and was regarded as adequate when bridging callus was seen at the fracture site in both AP and LAT views. Criteria of delayed union was kept at 3 months without any or very little evidence of callus formation and non union was deemed to have occurred if six months elapsed without any callus formation.

Functional assessment was done on basis of DASH score (Disabilities of the Arm,

Shoulder and Hand), ROM of wrist and forearm, Grip strength and Green and O'brien score.

ROM of wrist and forearm was measured at the time of suture removal and 4,12, 24 and 48 weeks post operatively and compared with the normal wrist. Similarly Grip strength was measured with Hand Dynamometer and compared with both injured and normal limb. DASH scoring was done at 12 weeks, 24 weeks and at one year post operatively.

Follow up was done at 4, 6, 24, 48 weeks post operatively and patient was assessed on the parameters as previously described.

The data was collected using a semi structured questionnaire. The ethical permission for conduction of this study was obtained from Ethical Committee of our institute. Written Informed consent were also taken from each participant after explaining them about this study.

\section{Results}

The age of study participants varied from 18 years to 73 years with mean age of 45.5 years and standard deviation of 19.5 years. Out of 80 patients included in the study 35 were male and 45 were female. About 2/3rd (65\%) of the participants came with injury of left hand while only 28 participants had right hand injury. Most common mode of injury was Road Traffic Accidents (52 patients) followed by fall on Outstretched Hand (28 patients).

Time from injury to surgery ranged between 2-12 days with mean duration of 5.40 days.

Surgery duration ranged from 50 to 100 minutes with a mean of 76.05 minutes and standard deviation of 17.01 minutes. Out of 80 patients, none of the patients developed infection, wound gaping, non-union, complex regional pain syndrome or tendon irritation. 6 out of 80 patients shown Wrist extension less than 20 degree. The mean preoperative radial inclination was 10.45 degrees while mean postoperative radial 
inclination was 18.1 degree. The difference was found to be statistically significant $(p<0.00)$. Similarly, the postoperative volar tilt and Radial Height was significantly more than preoperative volar tilt and Radial height, $p$-value $<0.00$. The range of movements have increased over the time period, the mean Wrist flexion at 6th week was $35.25^{\circ}$ which increased to $45.5^{\circ}$ on 12 th week and $51^{\circ}$ on 24 th postoperative week.

Mean Wrist extension on 6 th week was $24^{\circ}$ which changed to $37^{\circ}$ on 12 th and $42.5^{\circ}$ on $24^{\text {th }}$ postoperative week.

Mean pronation changed from $43.25^{\circ}$ on 6 th week to 54.25 on 12 th and 59.75 degrees on 24 th postoperative week.

Similarly, mean supination was found to be 59 degree on 6 th, 66 degree on 12th and 69.25 degree on 24th postoperative week.

The mean Grip Strength was 9.35 at 6 weeks, 18.35 at 12 weeks and 30.5 at 24 weeks and 48.25 at one year There was significant improvement in Grip Strength at 12 and 24 weeks as compared to 6 weeks with $p$-value $<0.00$. The baseline grip strength was taken to be grip strength found in normal limb at the time of admission. The minimum resumption of grip strength was $60 \%$ while maximum was $96.12 \%$.

The mean DASH score at 12 weeks was 9.41 and at one year was 7.2. The DASH score reduction was statistically significant in following week ( $p$ value $<0.00)$.

Around $12.5 \%$ of the patients showed poor Green and $\mathrm{O}$ ' Brien score at the end of 12 weeks $68.7 \%$ had a fair score and $18.7 \%$ had good score. The score improved at 24 weeks with $81.25 \%$ of patient having good Green and O' Brien score and rest showed fair score. At one year $93.7 \%$ of patients had a good score.

\section{Descriptive analysis of preoperative and postoperative radiological parameters of study participants}

\begin{tabular}{|c|c|c|c|c|c|}
\hline Parameters(n=80) & Minimum & Maximum & Mean & Std. Deviation & p-value \\
\hline pre-op radial inclination & 5 degree & 15 degree & 10.45 & 2.417 & 0.00 \\
\hline post-op radial inclination & 12 degree & 23 degree & 18.10 & 2.954 & \\
\hline pre-op volar tilt & -10 degree & 8 degree & 0.25 & 5.524 & 0.00 \\
\hline post-op solar tilt & 6 degree & 14 degree & 10.45 & 2.012 & \\
\hline pre-op radial height & $0 \mathrm{~mm}$ & $8 \mathrm{~mm}$ & 3.20 & 2.441 & 0.00 \\
\hline post-op radial height & $4 \mathrm{~mm}$ & $12 \mathrm{~mm}$ & 9.25 & 2.268 & \\
\hline
\end{tabular}

Descriptive analysis of postoperative Grip strength (GS) and DASH scores of study participants

\begin{tabular}{|c|c|c|c|c|c|}
\hline Parameter (weeks) & Minimum & Maximum & Mean & Std. Deviation & p-value \\
\hline Grip strength 6wks & $3 \mathrm{~kg}$ & $20 \mathrm{~kg}$ & 9.35 & 4.955 & 0.00 \\
\hline Grip strength 12 wks & $8 \mathrm{~kg}$ & $30 \mathrm{~kg}$ & 18.35 & 7.936 & \\
\hline Grip strength $24 \mathrm{wks}$ & $12 \mathrm{~kg}$ & $55 \mathrm{~kg}$ & 30.50 & 15.143 & 0.00 \\
\hline Grip strength $1 \mathrm{yr}$ & $28 \mathrm{~kg}$ & $57 \mathrm{~kg}$ & 48.25 & 22.6 & \\
\hline DASH score 12 wks & 6.4 & 14.5 & 9.410 & 2.0624 & \\
\hline DASH score 24 wks & 4.5 & 11.2 & 7.485 & 2.0270 & 0.00 \\
\hline DASH score $1 \mathrm{yr}$ & 4 & 10.3 & 7.2 & 1.82 & \\
\hline
\end{tabular}

\section{Grading according to Green and O'Brien score at 12 and 24 weeks postoperatively}

\begin{tabular}{|c|c|c|}
\hline Green and O'Brien score results & 12 weeks & 24 weeks \\
\hline POOR & 10 & 0 \\
\hline FAIR & 55 & 0 \\
\hline GOOD & 15 & 5 \\
\hline
\end{tabular}

\section{Discussion}

Minimally invasive plate osteosynthesis has been used in management of fractures of various long bones. MIPO provides the advantage of less soft tissue dissection and periosteal stripping as compared to conventional ORIF. The advantages of MIPO can provide better outcomes in management of distal radius fractures and with this case series we have tried to study this.

Distal radius fractures if not managed adequately by proper reduction of articular surface and a stable rigid fixation can lead to a host of complications at the wrist. After managing the patients by minimally invasive technique we followed them for complications and saw their functional outcomes.

The mean age of patients in our study was 42.55 years. This was more or less in accordance with a studies done by Rajeev Shukla et al. ${ }^{[5]}$, and Ashish N. Chauhan et al. ${ }^{[6]}$ who found the found mean age group of distal radius fracture to be 39.12 years and 42.8 years showing that these injuries are most common in late thirties and early forties. By this we inferred that young or individuals of active age group are more prone for sustaining these fractures.
In our case series majority of patients sustained trauma due to road traffic accidents followed by fall on outstretched hand showing this to be the most common mechanism of distal end radius fractures.

Various modalities for treatment of distal radius fractures have been studied like ORIF with plate and screws, external fixator application or fixation by k-wires. Wright et al. ${ }^{\text {[7] }}$ followed distal radius fractures managed by plating and ext. fix. Application and found no functional difference between the patients managed by either modality. Another study by Egol et al. ${ }^{[8]}$ documented improved results initially in patients managed by plating however at final followup the functional outcome was almost same in patients of either group.

External fixators in distal radius fractures are associated with increased incidence of infections and stiffness due to poor articular reduction where as open reduction and plating can cause greater soft tissue dissection leading to soft tissue related problems. Both these issues are not encountered in MIPO.

Comparable results as ours were obtained in studies done by X.-M Wei et al. ${ }^{[9]}$, Atin Kumar Kundu et al. ${ }^{[10]}$ and Alvin 
Chao Yu Chen et al. ${ }^{[11]}$.

Xiaofei Yu et al. ${ }^{[12]}$ did a comparative study and reported an infection rate of $33.3 \%$ in external fixator group and $7.7 \%$ in volar locking plate group however we did not encounter any incidences of infection in the followup of our patients.

The functional outcome of our study was quite satisfactory and we can even say better than other studies that evaluated open reduction and fixator application for distal radius fractures. This was evidenced by the comparison of our DASH score values at 3,6 and 12 months of followup with other studies like that of Lee et al. ${ }^{[13]}$. Lee et al. documented a DASH score of 10.1 whereas our study showed a DASH score consistently less than 10 right from the beginning.

One problem with the MIPO technique for distal radius fracture fixation that has been stated by a few is the inadvertent injury to the median nerve, its palmar cutaneous branch or sometimes the radial artery as the exposure done is quite minimal. However in our study we were vigilant about the safety zones and carefully followed the relations of the nerve and artery to the FCR tendon and avoided any injury to them. Two patients in immediate post-op period complained of tingling along the index finger but it disappered by the end of its week post surgery.

Our study has few limitations as we had a big enough sample size of 80 patients and our duration of followup was also almost two and half years which is adequate. It was a bit difficult to achieve a sample size of 80 with a inclusion criteria of minimal intra articular communition but over a duration of two and a half years and with the inclusion of a few retrospective cases it was achieved. The only drawback we had was that there was no control group who were managed with open plating by Henry's approach. Other strengths of our study includes single investigator to eliminate observer bias and there was no loss to follow up.

\section{Conclusion}

Minimally invasive plate osteosynthesis can be utilised for extra articuar distal radius fracture and intra articular fractures with minimal communition. Minimally invasive plate osteosynthesis avoids extensive soft tissue dissection, preserves the pronator quadratus and the periosteal blood supply of the distal radius. It provides rigid fixation and allows early mobilisation thus giving better clinical, radiological and functional outcomes. Literally no complications and good functional results and reduced hospital stays have proven Minimally invasive plate osteosynthesis to be a better treatment option for fracture distal end radius.

\section{References}

1. Kumar. Distal Radius Fracture managed by minimally invasive plate osteosynthesis. IJSS 2016;(4):161

2. Kapoor H, Agarwal A, Dhaon B. Displaced intraarticular fractures of distal radius: A comparative evaluation of results following closed reduction, external fixation and open reduction with internal fixation. Injury 2000;31:759.

3. Kreder H, Hanel D, Agel J, McKee M, Schemitsch E, Trumble $\mathrm{T}$ et al.. Indirect reduction and percutaneous fixation versus open reduction and internal fixation for displaced intraarticular fractures of the distal radius: A randomised, controlled trial. J Bone Joint Surg $\mathrm{Br}$ 2005;87:829-36.

4. Helfet DL, Shonnard PY, Levine D, Borrelli J. Minimally invasive plate osteosynthesis of distal fractures of the tibia. Injury 1997;28:A42-8.

5. Shukla R, Jain RK, Sharma NK, Kumar R. External fixator versus volar locking plate for displaced intra articular distal radius fractures: a prospective Randomized comparative study of the functional outcomes. J Orthop Traumatol 2014;15(4):265-270.

6. Ashish N. Functional and radiological outcome in distal radius fractures treated with locking compression plate. Int J Res Med Sci 2017;5(2):574-582.

7. Wright TW, Horodyski M, Smith DW. Functional outcome of unstable distal radius fractures: ORIF with a volar fixed angle tine plate versus external fixation. J Hand Surg Am 2005;30:289-99.

8. Egol K, Walsh M, Tejwani N, McLaurin T, Wynn C, Paksima N. Bridging external fixation and supplementary Kirschner-wire fixation versus volar locked plating for unstable fractures of the distal radius: A randomised, prospective trial. J Bone Joint Surg Br 2008;90:1214-21.

9. Wei XM, Sun ZZ, Rui YJ, Song XJ, Jiang WM. Minimally invasive percutaneous plate osteosynthesis for distal radius fractures with long segment metadisphyseal communition. Orthop Traumatol Surg Res 2016;102(3):333-8.

10. Kundu AK. Intra articular distal radius fracture and volar plate fixation: a prospective study. Int J Res Orthop 2017;3(3):589-594.

11. Chen ACY, Chou YC, Cheng CY. Distal radius fractures: Minimal invasive plate osteosynthesis with dorsal bicolumnar locking plates fixation. Indian J Orthop 2017;51(1):93-8.

12. Xiaofei Yu, Yu Y, Shao X, Bai Y, Zhou T. eVolar locking plate versus external fixation with optional additional $\mathrm{K}$-wire for treatment of $\mathrm{AO}$ type $\mathrm{C} 2 / \mathrm{C} 3$ fractures: a retrospective comparative study. J Orthop Surg Res 2019;14:271.

13. Lee SK, Seo DW, Kim KJ, Yang DS, Choy WS. Volar long locking compression plate fixation for distal radius fractures with metaphyseal and diaphyseal extension Eur J Orthop Surg Traumatol 2013;23:407-415. 